\title{
Bifurcation Analysis of a Chemostat Model of Plasmid-Bearing and Plasmid-Free Competition with Pulsed Input
}

\author{
Zhong Zhao, Baozhen Wang, Liuyong Pang, and Ying Chen \\ Department of Mathematics, Huanghuai University, Zhumadian, Henan 463000, China \\ Correspondence should be addressed to Zhong Zhao; zhaozhong8899@163.com
}

Received 18 April 2014; Revised 17 May 2014; Accepted 24 May 2014; Published 15 June 2014

Academic Editor: Mohamad Alwash

Copyright (c) 2014 Zhong Zhao et al. This is an open access article distributed under the Creative Commons Attribution License, which permits unrestricted use, distribution, and reproduction in any medium, provided the original work is properly cited.

\begin{abstract}
A chemostat model of plasmid-bearing and plasmid-free competition with pulsed input is proposed. The invasion threshold of the plasmid-bearing and plasmid-free organisms is obtained according to the stability of the boundary periodic solution. By use of standard techniques of bifurcation theory, the periodic oscillations in substrate, plasmid-bearing, and plasmid-free organisms are shown when some conditions are satisfied. Our results can be applied to control bioreactor aimed at producing commercial producers through genetically altered organisms.
\end{abstract}

\section{Introduction}

Biofermentation has become an active area of research on the continuous cultivation of microorganism in recent years [13 ]. The chemostat is an important laboratory apparatus used to continuously culturing microorganisms [2-8]. It can be used to investigate microbial growth because the parameters are measurable, the experiments are reasonable, and the mathematics is tractable [9].

Fermentations using genetically modified (recombinant) microorganisms typically contain two kinds of cellsrecombinant cells and wild-type cells. The former contains a genetically inserted plasmid (a foreign DNA molecule that can exist independent of the host chromosome and can replicate autonomously) which is responsible for the coding functions that result in the synthesis of a desired protein. Wild-type or plasmid-free cells do not contain this plasmid and therefore cannot generate the protein. Nevertheless, they consume nutrients, grow, and multiply. From this perspective, plasmid-free cells may thus be considered undesirable, and different methods are employed to check their proliferation. As recombinant (or plasmid-bearing) cells have to support a larger metabolic load than plasmid-free cells, their growth rates are smaller. In addition, these cells lose their plasmids during the fermentation process.
With the scientific technology, the importance of the genetically altered technology is widely recognized. Therefore, it is necessary to understand the dynamic behavior of the fermentation process. Xiang and Song [10] analyze a simple chemostat model for plasmid-bearing and plasmid-free organisms with the pulsed substrate and linear functional response. They prove that system is permanent if the impulsive period is less than some critical value. Shi et al. [11] consider a new Monod type chemostat model with delayed growth response and pulsed input in the polluted environment. Normally, the velocity of the enzyme reaction increases with the increase in substrate concentration. Some enzymes, however, display the phenomenon of excess substrate inhibition, which means that large amounts of substrate can have the adverse effect and actually slow the reaction down. The Monod function does not account for any inhibitory effect at high substrate concentration. Therefore, it is crucial to choose a response function showing the excess substrate inhibition. Pal et al. [12] introduce the MonodHaldene functional responses into a three-tier model of phytoplankton, zooplankton, and nutrient in order to investigate the phenomenon of excess substrate inhibition. Therefore, we introduce the Monod-Haldene functional 
response into the following chemostat model with pulsed input:

$$
\begin{gathered}
\dot{S}(\bar{t})=-Q S-\frac{\mu_{1} x_{1} S}{\delta\left(A+S+B S^{2}\right)}-\frac{\mu_{2} x_{2} S}{\delta\left(A+S+B S^{2}\right)}, \\
\dot{x}_{1}(\bar{t})=x_{1}\left(\frac{\mu_{1}(1-q) S}{A+S+B S^{2}}-Q\right), \\
\dot{x}_{2}(\bar{t})=x_{2}\left(\frac{\mu_{2} S}{A+S+B S^{2}}-Q\right)+\frac{q \mu_{1} S x_{1}}{A+S+B S^{2}}, \\
\bar{t} \neq \frac{n \tau}{Q}, \\
\Delta S=S^{0} \tau, \\
\Delta x_{1}=0, \quad \bar{t}=\frac{n \tau}{Q}, \\
\Delta x_{2}=0,
\end{gathered}
$$

where $\Delta S=S\left(n \tau^{+} / Q\right)-S(n \tau / Q), \Delta x_{1}=x_{1}\left(n \tau^{+} / Q\right)-$ $x_{1}(n \tau / Q), \Delta x_{2}=x_{2}\left(n \tau^{+} / Q\right)-x_{2}(n \tau / Q)$, and $n \in Z_{+}, Z_{+}=$ $\{1,2, \ldots\} . A(A>0)$ can be interpreted as the halfsaturation constant in the absence of any inhibitory effect. $B(B>0)$ is the measure of inhibitory effect. $S(\bar{t})$ denotes the nutrient concentration at time $\bar{t}$, and $x_{1}(\bar{t})$ denotes the concentration of plasmid-bearing organism at time $\bar{t} . x_{2}(\bar{t})$ is the concentration of plasmid-free organism at time $\bar{t} . \mu_{1}$ and $\mu_{2}$ are the uptake constant of the microorganism. $\delta$ is the constant yield (It is reasonable to assume that the yield constants for two organisms are the same since they are the same organism just with or without the plasmid). $S^{0}$ represents the input concentration of the nutrient each time, and the probability that a plasmid is lost in reproduction is represented by $q(0<q<1)$. $Q(0<Q<1)$ is the washout proportion of the chemostat each time. $n \tau / Q$ is the period of the pulse.

The variables in the above system may be rescaled by measuring $x(t) \equiv S(\bar{t}) / S^{0}, y(t) \equiv x_{1}(\bar{t}) / \delta S^{0}, z(t) \equiv x_{2}(\bar{t}) / S^{0} \delta$, and $t=Q \bar{t}$, and then we have the following system:

$$
\begin{aligned}
& \dot{x}(t)=-x-\frac{m_{1} x y}{a_{1}+x+a_{2} x^{2}}-\frac{m_{2} x z}{a_{1}+x+a_{2} x^{2}}, \\
& \dot{y}(t)=y\left(\frac{m_{1}(1-q) x}{a_{1}+x+a_{2} x^{2}}-1\right), \\
& \dot{z}(t)=z\left(\frac{m_{2} x}{a_{1}+x+a_{2} x^{2}}-1\right)+\frac{m_{1} q x y}{a_{1}+x+a_{2} x^{2}}, \\
& t \neq n \tau,
\end{aligned}
$$

$$
\begin{aligned}
& \Delta x=\tau, \\
& \Delta y=0, \quad t=n \tau, \\
& \Delta z=0,
\end{aligned}
$$

where $m_{1}=\mu_{1} / Q, m_{2}=\mu_{2} / Q, a_{1}=A / S^{0}, a_{2}=B S^{0}$.

\section{The Behavior of the Substrate and Plasmid-Free Organism Subsystem}

In the absence of the plasmid-bearing organism, system (2) is reduced to

$$
\begin{gathered}
\dot{x}(t)=-x-\frac{m_{2} x z}{a_{1}+x+a_{2} x^{2}}, \\
\dot{z}(t)=z\left(\frac{m_{2} x}{a_{1}+x+a_{2} x^{2}}-1\right), \\
\Delta x=\tau, \quad t=n \tau . \\
\Delta z=0, \quad t=
\end{gathered}
$$

This nonlinear system has a simple periodic solution. For our purpose, we present the solution in this section.

We add the first and the second equations of (3). We have

$$
\frac{d(x+z)}{d t}=-(x+z) \text {. }
$$

Taking the variable change $s=x+y$, the system (3) can be rewritten as

$$
\begin{gathered}
\frac{d s}{d t}=-s, \quad t \neq n \tau, \\
s\left(n \tau^{+}\right)=s(n \tau)+\tau, \quad t=n \tau .
\end{gathered}
$$

Thus, we have the following.

Lemma 1. System (5) has a positive periodic solution $\widetilde{\boldsymbol{s}}(t)$ and for any solution $s(t)$ of system $(5)|s(t)-\widetilde{s}(t)| \rightarrow 0$ as $t \rightarrow \infty$, where $\widetilde{s}(t)=(\tau \exp (-(t-n \tau)) /(1-\exp (-\tau))), t \in(n \tau,(n+1) \tau]$, and $\widetilde{s}\left(0^{+}\right)=\tau /\left(1-e^{-\tau}\right)$.

Proof. Clearly, $\widetilde{\boldsymbol{s}}(t)$ is a positive periodic solution of the system (5). Any solution $s(t)$ of system (5) is $s(t)=(s(0)-(\tau /(1-$ $\left.\left.\left.e^{-\tau}\right)\right)\right) e^{-t}+\widetilde{\mathcal{S}}(t), t \in(n \tau,(n+1) \tau], n \in Z_{+}$. Hence, $|s(t)-\tilde{s}(t)| \rightarrow$ 0 as $t \rightarrow \infty$.

Lemma 2. Let $(x(t), z(t))$ be any solution of system (5) with initial condition $x(0) \geq 0, z(0)>0$, and then $\lim _{t \rightarrow \infty} \mid x(t)+$ $z(t)-\widetilde{s}(t) \mid=0$.

Lemma (5) shows that the periodic solution $\widetilde{s}(t)$ is uniquely invariant manifold of system (3). Therefore, one has $0<x(t) \leq$ $M, 0<z(t) \leq M$, where $M=\tau /\left(1-e^{-T}\right)$.

Theorem 3. For system (3), one has the following.

(1) If $(1 / \tau) \int_{0}^{\tau}\left(m_{2} \widetilde{s}(l) /\left(a_{1}+\widetilde{s}(l)+a_{2} \widetilde{s}(l)^{2}\right)\right) d l<1$, system (3) has a uniquely globally stable boundary $\tau$-periodic solution $\left(x_{e}(t), z_{e}(t)\right)$, where $x_{e}(t)=\widetilde{s}, z_{e}(t)=0$.

(2) If $(1 / \tau) \int_{0}^{\tau}\left(m_{2} \widetilde{s}(l) /\left(a_{1}+\widetilde{s}(l)+a_{2} \widetilde{s}(l)^{2}\right)\right) d l>1$, system (3) has a globally asymptotically stable positive $\tau$-periodic solution $\left(x_{s}(t), z_{s}(t)\right)$, and one has

$$
\int_{0}^{\tau} \frac{m_{2}\left(\widetilde{s}(t)-z_{s}(t)\right)}{a_{1}+\widetilde{s}(t)-z_{s}(t)+a_{2}\left(\widetilde{s}(t)-z_{s}(t)\right)^{2}} d t=1 .
$$


Proof. (1) If $(1 / \tau) \int_{0}^{\tau}\left(m_{2} \widetilde{s}(l) /\left(a_{1}+\widetilde{s}(l)+a_{2} \widetilde{s}(l)^{2}\right)\right) d l<1$, it is obvious that

$$
\begin{aligned}
z(t) \leq & z(0) \exp \left(\left(\frac{1}{\tau} \int_{0}^{\tau} \frac{m_{2} \widetilde{s}(l)}{a_{1}+\widetilde{s}(l)+a_{2} \widetilde{s}(l)^{2}} d l-1\right) t\right) \\
& \times \exp \left(\int_{0}^{t} p_{1}(l) d l\right),
\end{aligned}
$$

where $p_{1}(t)=\left(m_{2} \widetilde{s}(l) /\left(a_{1}+\widetilde{s}(l)+a_{2} \widetilde{s}(l)^{2}\right)\right)-$ $(1 / \tau) \int_{0}^{\tau}\left(m_{2} \widetilde{s}(l) /\left(a_{1}+\widetilde{s}(l)+a_{2} \widetilde{s}(l)^{2}\right)\right) d l . p_{1}(t)$ is $\tau$-periodic piecewise continuous function in view of $(1 / \tau) \int_{0}^{\tau} p_{1}(l) d l=0$. For $(1 / \tau) \int_{0}^{\tau}\left(m_{2} \widetilde{s}(l) /\left(a_{1}+\widetilde{s}(l)+a_{2} \widetilde{s}(l)^{2}\right)\right) d l-1<0$, we obtain $z(t)$ that tends exponentially to zero as $t \rightarrow+\infty$. From Lemma 2 and system (5), we have $\lim _{t \rightarrow \infty}|x(t)-\widetilde{s}(t)|=0$.

(2) If $(1 / \tau) \int_{0}^{\tau}\left(m_{2} \widetilde{s}(l) /\left(a_{1}+\widetilde{s}(l)+a_{2} \widetilde{s}(l)^{2}\right)\right) d l>1$, we consider system (3) in its stable invariant manifold $\widetilde{s}(t)$; that is,

$$
\begin{array}{r}
\frac{d z}{d t}=\frac{m_{2}(\widetilde{s}(t)-z(t)) z(t)}{a_{1}+\widetilde{s}(t)-z(t)+a_{2}(\widetilde{s}(t)-z(t))^{2}}-z, \\
0 \leq z_{0} \leq \widetilde{s}(0) .
\end{array}
$$

Suppose $z\left(t, z_{0}\right)$ is a solution of (8) with initial condition $z_{0} \in$ $(0, \widetilde{s}(0)]$, and we obtain

$$
\begin{aligned}
& z\left(t, z_{0}\right) \\
& =z(n \tau) \exp \left(\int _ { n \tau } ^ { t } \left(\frac{m_{2}(\widetilde{s}(t)-z(t))}{a_{1}+\widetilde{s}(t)-z(t)+a_{2}(\widetilde{s}(t)-z(t))^{2}}\right.\right. \\
& -1) d l) \\
& z(n \tau)=z_{0}, \quad t \in(n \tau,(n+1) \tau]
\end{aligned}
$$

For (9), we have the following properties:

(i) the function $F\left(z_{0}\right)=z\left(t, z_{0}\right), z_{0} \in(0, \widetilde{s}(0)]$ is an increasing function;

(ii) $0<z\left(t, z_{0}\right) z<\widetilde{s}(0), t \in[0, \infty)$ is a continuous function;

(iii) $z(t, 0)=0, t \in[0, \infty)$ is a solution.

The periodic solution of (9) satisfies the following equation:

$$
\begin{aligned}
& z_{0} \\
& =z_{0} \exp \left(\int_{0}^{\tau}\left(\frac{m_{2}(\widetilde{s}(l)-z(l))}{a_{1}+\widetilde{s}(l)-z(l)+a_{2}(\widetilde{s}(l)-z(l))^{2}}-1\right) d l\right) .
\end{aligned}
$$

And we denote $m_{2}^{*}=\left(\tau / \int_{0}^{\tau}\left(\widetilde{s}(l) /\left(a_{1}+\widetilde{s}(l)+a_{2} \widetilde{s}(l)^{2}\right)\right) d l\right)$. By (i) and (ii), system (8) has a stable solution $z_{e}(t)=0$ for $m_{2}<$ $m_{2}^{*}$. By Lemma 2, we have $\lim _{t \rightarrow \infty}|x(t)-\widetilde{s}(t)|=0$.
If $m_{2}>m_{2}^{*}$, system (8) has a uniquely positive periodic solution. We denote the positive periodic solution

$$
z_{s}(t)=z\left(t, z_{0}^{*}\right), \quad x_{s}(t)=\widetilde{s}(t)-z\left(t, z_{0}^{*}\right) .
$$

From (9), we obtain

$$
\int_{0}^{\tau} \frac{m_{2}\left(\widetilde{s}(t)-z_{s}(t)\right)}{a_{1}+\widetilde{s}(t)-z_{s}(t)+a_{2}\left(\widetilde{s}(t)-z_{s}(t)\right)^{2}} d t=1,
$$

where $z_{0}^{*}=z_{s}(0)$.

In order to prove the stability of the periodic solution $z_{s}(t)$, we define a function $F:\left(t, z_{0}\right) \rightarrow R \in[0, \infty) \times[0, \widetilde{s}(0)]$ as the following:

$$
F\left(z\left(t, z_{0}\right)\right)=\int_{0}^{t} \frac{m_{2}\left(\widetilde{s}(t)-z_{s}(t)\right)}{a_{1}+\widetilde{s}(t)-z_{s}(t)+a_{2}\left(\widetilde{s}(t)-z_{s}(t)\right)^{2}} d t-t .
$$

Noticing (8), we have

$$
F\left(z\left(\tau, z_{0}\right)\right)=\ln \left(\frac{z\left(\tau, z_{0}\right)}{z_{0}}\right), \quad z_{0} \in(0, \widetilde{s}(0)],
$$

and it is obvious that $F\left(z(n \tau), z_{0}^{*}\right)=0$. For any $z_{0} \in(0, \widetilde{s}(0)]$, by the theorem on the differentiability of the solutions on the initial values, $\partial z\left(t, z_{0}\right) / \partial z_{0}$ exists. Furthermore, $\partial z\left(t, z_{0}\right) / \partial z_{0}$ holds for $t \in(0, \infty)$ (otherwise, there exists $0<z_{1}<$ $z_{2}<\widetilde{s}(0)$ such that $z\left(t_{0}, z_{1}\right)=z\left(t_{0}, z_{2}\right)$ for $t_{1}>0$, which is a contradiction to the different flows of system (8) not to intersect, and we have $\tilde{s}(l)>z\left(l, z_{0}\right)$ for $l \in[0, \tau]$. So, we obtain that

$$
\frac{d F\left(z\left(\tau, z_{0}\right)\right)}{d z_{0}}<0 .
$$

Therefore, $F\left(z\left(\tau, z_{0}\right)\right)$ is monotonously decreasing continuous function for $z_{0} \in[0, \widetilde{s}(0)]$. Now, we choose $\varepsilon$ such as $0<\varepsilon<z_{0}^{*}<\widetilde{s}(0)$, and we have the following cases:

$$
\begin{gathered}
\ln z\left(\tau, z_{0}\right)-\ln z_{0}<0, \quad \text { if } z_{0}^{*}<z_{0}<\tilde{s}(0), \\
\ln z\left(\tau, z_{0}\right)-\ln z_{0}=0, \quad \text { if } z_{0}^{*}=z_{0}, \\
\ln z\left(\tau, z_{0}\right)-\ln z_{0}>0, \quad \text { if } \varepsilon<z_{0}<z_{0}^{*} .
\end{gathered}
$$

Furthermore, we obtain the following equations:

$$
\begin{gathered}
z_{0}>z\left(\tau, z_{0}\right)>\cdots z\left(n \tau, z_{0}\right)>z_{0}^{*}, \quad \text { if } z_{0}^{*}<z_{0} \leq \widetilde{s}(0), \\
z_{0}<z\left(\tau, z_{0}\right)<\cdots z\left(n \tau, z_{0}\right)<z_{0}^{*}, \quad \text { if } \varepsilon<z_{0}<z_{0}^{*} .
\end{gathered}
$$

Suppose that

$$
\lim _{n \rightarrow \infty} z\left(n \tau, z_{0}\right)=a
$$

We will prove that the solution $z(t, a)$ is $\tau$-periodic. Because the function $z_{n}(t)=z\left(t+n \tau, z_{0}\right)$ is also a solution of system (8) and $z_{n}(0) \rightarrow a$ as $n \rightarrow \infty$, we have that $z(\tau, a)=\lim _{n \rightarrow \infty} z_{n}(\tau)=a$ by the continuous dependence of 
the solutions on the initial values. Hence, the solution $z(t, a)$ is $\tau$-periodic. The periodic solution $z\left(t, z_{0}^{*}\right)$ is unique and $a=z_{0}^{*}$. Let $\varepsilon>0$ be given, by the theorem on the continuous dependence of the solutions on the initial values, there exists a $\delta>0$ such that

$$
\left|z\left(t, z_{0}\right)-z\left(t, z_{0}^{*}\right)\right|<\varepsilon,
$$

if $\left|z_{0}-z_{0}^{*}\right|<\delta$ and $0 \leq t \leq \tau$. Choose $n_{1}>0$ such as $\left|z\left(n \tau, z_{0}\right)-z_{0}^{*}\right|<\delta$ for $n>n_{1}$. Then, $\left|z\left(t, z_{0}\right)-z\left(t, z_{0}^{*}\right)\right|<\varepsilon$ for $t>n \tau$, which shows that

$$
\lim _{n \rightarrow \infty}\left|z\left(t, z_{0}\right)-z\left(t, z_{0}^{*}\right)\right|=0, \quad z_{0} \in(0, \widetilde{s}(0)] .
$$

For system (3), we obtain $\lim _{t \rightarrow \infty}\left|x-x_{s}\right|=0$ and $\lim _{t \rightarrow \infty} \mid z-$ $z_{s} \mid=0$ for any solution $(x(t), z(t))$ with the initial condition $x(0) \geq 0, z(0)>0$. From the $\tau$-periodic solution $z_{s}$ being asymptotically stable, we obtain the multiplier $\mu$ of $z_{s}$, which satisfies

$$
\mu=\exp \left(\int_{0}^{\tau} \frac{m_{2} x_{s}(l)\left(a_{1}-a_{2} x_{s}^{2}(l)\right)}{\left(a_{1}+x_{s}(l)+a_{2} x_{s}^{2}(l)\right)^{2}} d l\right)<1 .
$$

This conclusion will be used in Section 3.

\section{The Bifurcation of the System}

In order to investigate the properties of system (2), we add the first, second, and third equations of system (2) and take variable change $s=x+y+z$, and the following lemma is obvious.

Lemma 4. Let $X(t)=(x(t), y(t), z(t))$ be any solution of system (2) with $X(0)>0$, and then

$$
\lim _{t \rightarrow \infty}|x(t)+y(t)+z(t)-\widetilde{s}(t)|=0 .
$$

For convenience, we suppose $m_{2}>m_{2}^{*}$ and denote that

$$
m_{1}^{*}=\frac{\tau}{(1-q) \int_{0}^{\tau}\left(z_{s}(l) /\left(a_{1}+z(l)+a_{2} z_{s}^{2}(l)\right)\right) d l} .
$$

Theorem 5. Let $(x(t), y(t) z(t))$ be any solution of system (2) with $X(0)>0$, and we obtain the following.

(1) If $m_{2}<m_{2}^{*}$, then system (2) has a unique globally asymptotically stable boundary $\tau$-periodic solution $(\widetilde{s}(t), 0,0)$.

(2) If $m_{2}>m_{2}^{*}$ and $m_{1}<m_{1}^{*}$, then system (2) has a unique globally asymptotically stable boundary $\tau$-periodic solution $\left(x_{s}(t)\right), 0, z_{s}(t)$.

(3) If $m_{2}>m_{2}^{*}$ and $m_{1}>m_{1}^{*}$, then boundary $\tau$-periodic solution $\left(x_{s}(t)\right), 0, z_{s}(t)$ of system (2) is unstable.

Proof. The proof of (1) is easy; we want to prove (2) and (3). The local stability of periodic solution $\left(x_{s}(t), 0, z_{s}(t)\right)$ may be determined by considering the behavior of small amplitude of the solution. Define

$$
x(t)=w(t)+x_{s}(t), \quad y(t)=v(t), \quad z(t)=w(t)+z_{s}(t) .
$$

There may be written

$$
\left(\begin{array}{c}
u(t) \\
v(t) \\
w(t)
\end{array}\right)=\Phi(t)\left(\begin{array}{c}
u(0) \\
v(0) \\
w(0)
\end{array}\right), \quad 0 \leq t \leq \tau,
$$

where $\Phi(t)=\left\{\varphi_{i j}\right\}_{i, j=1,2,3}$ satisfies

$$
\frac{d \Phi(t)}{d t}=\left(\begin{array}{ccc}
-1-\frac{m_{2}\left(a_{1}-a_{2} x_{s}\right) z_{s}}{\left(a_{1}+x_{s}+a_{2} x_{s}^{2}\right)^{2}} & -\frac{m_{1} x_{s}}{a_{1}+x_{s}+a_{2} x_{s}^{2}} & -\frac{m_{2} x_{s}}{a_{1}+x_{s}+a_{2} x_{s}^{2}} \\
\frac{m_{2}\left(a_{1}-a_{2} x_{s}\right) z_{s}}{\left(a_{1}+x_{s}+a_{2} x_{s}^{2}\right)^{2}} & -1+\frac{m_{2} x_{s}}{a_{1}+x_{s}+a_{2} x_{s}^{2}} & \frac{m_{1} q x_{s}}{a_{1}+x_{s}+a_{2} x_{s}^{2}} \\
0 & 0 & -1+\frac{m_{1}(1-q) x_{s}}{a_{1}+x_{s}+a_{2} x_{s}^{2}}
\end{array}\right) \Phi(t),
$$

and $\Phi(0)=I$ is the identity matrix. Hence, the fundament solution matrix is

$$
\Phi(\tau)=\left(\begin{array}{ccc}
\varphi_{11}(\tau) & \varphi_{12}(\tau) & * \\
\varphi_{21}(\tau) & \varphi_{22}(\tau) & * * \\
0 & 0 & \exp \left(\int_{0}^{\tau}\left(\frac{m_{1}(1-q) x_{s}(l)}{a_{1}+x_{s}(l)+a_{2} x_{s}^{2}(l)}-1\right) d l\right)
\end{array}\right)
$$


and there is no need to calculate the exact form $(*)$ and $* *$ as it is not required in the analysis that follows.

The eigenvalues of the matrix $\Phi(\tau)$ are $\mu_{3}=$ $\exp \left(\int_{0}^{\tau}\left(\left(m_{1}(1-q) x_{s}(l) /\left(a_{1}+x_{s}(l)+a_{2} x_{s}^{2}(l)\right)\right)-1\right) d l\right), \mu_{1}$, and $\mu_{2}$, where $\mu_{1}$ and $\mu_{2}$ are determined by the following matrix:

$$
\Phi(\tau)=\left(\begin{array}{ll}
\varphi_{11}(\tau) & \varphi_{12}(\tau) \\
\varphi_{21}(\tau) & \varphi_{22}(\tau)
\end{array}\right)
$$

$\mu_{1}$ and $\mu_{2}$ are also the multipliers of the local linearization system of (3). According to Theorem 3, we have that $\mu_{1}<1$ and $\mu_{2}<1$.

If $m_{2}>m_{2}^{*}$ and $m_{1}<m_{1}^{*}$ and $\mu_{3}=\exp \left(\int_{0}^{\tau}\left(\left(m_{1}(1-\right.\right.\right.$ q) $\left.\left.\left.x_{s}(l) /\left(a_{1}+x_{s}(l)+a_{2} x_{s}^{2}(l)\right)\right)-1\right) d l\right)<1$, the boundary periodic solution $\left(x_{s}(t), 0, z_{s}(t)\right)$ of system (2) is locally asymptotically stable. In view of $y(t) \leq y(0) \exp \left(\int_{0}^{t}\left(\left(m_{1}(1-\right.\right.\right.$ $\left.\left.\left.q) x_{s}(l) /\left(a_{1}+x_{s}(l)+a_{2} x_{s}^{2}(l)\right)\right)-1\right) d l\right)$, we obtain $y(t) \rightarrow 0$ as $t \rightarrow \infty$.

From $\lim _{t \rightarrow \infty}|x(t)+y(t)+z(t)-\widetilde{s}(t)|=0$, we have $\lim _{t \rightarrow \infty}|x(t)+z(t)-\widetilde{s}(t)|=0$. Now, using Theorem 3 , we have $\lim _{t \rightarrow \infty}\left|z(t)-\widetilde{z}_{s}(t)\right|=0$ and $\lim _{t \rightarrow \infty}\left|x(t)-\tilde{x}_{s}(t)\right|=0$.

If $m_{2}>m_{2}^{*}, m_{1}>m_{1}^{*}$ and $\mu_{3}=\exp \left(\int_{0}^{\tau}\left(\left(m_{1}(1-\right.\right.\right.$ q) $\left.\left.\left.x_{s} /\left(a_{1}+x_{s}+a_{2} x_{s}^{2}\right)\right)-1\right) d l\right)>1$, the boundary periodic solution $\left(x_{s}(t), 0, s_{s}(t)\right)$ of system (2) is unstable. The proof is completed.

Let $B$ denote the Banach space of continuous, $\tau$-periodic function $N:[0, \tau] \rightarrow R^{2}$. In the set $B$, we introduce the norm $|N|_{0}=\sup _{0 \leq t<\tau}|N(t)|$ with which $B$ becomes a Banach space with the uniform convergence topology. For convenience, just like [13], we introduce the following Lemmas 6 and 7.

Lemma 6. Suppose $a_{i j} \in B$.

(a) If $\int_{0}^{\tau} a_{22}(s) d s \neq 0, \int_{0}^{\tau} a_{11}(s) d s \neq 0$, then the linear homogenous system

$$
\begin{gathered}
\frac{d y_{1}}{d t}=a_{11} y_{1}+a_{12} y_{2}, \\
\frac{d y_{2}}{d t}=a_{22} y_{1},
\end{gathered}
$$

has no nontrivial solution in $B \times B$. In this case, the nonhomogeneous system

$$
\begin{gathered}
\frac{d y_{1}}{d t}=a_{11} y_{1}+a_{12} y_{2}+f_{1}, \\
\frac{d y_{2}}{d t}=a_{22} y_{1}+f_{2},
\end{gathered}
$$

has, for every $\left(f_{1}, f_{2}\right) \in B \times B$, a unique solution $\left(x_{1}, x_{2}\right) \in$ $B \times B$ and the operator $L: B \times B \rightarrow B \times B$ defined by $\left(x_{1}, x_{2}\right)=$ $L\left(f_{1}, f_{2}\right)$ is linear and compact. If we define that $x_{2}^{\prime}=a_{22} x_{2}+f_{2}$ has a unique solution $x_{2} \in B$ and the operator $L_{2}: B \rightarrow B$ defined by $x_{2}=L_{2} f_{2}$ is linear and compact. Further, $x_{1}^{\prime}=$ $a_{11} x_{1}+f_{3}\left(f_{3} \in B\right)$ has a unique solution (since $\int_{0}^{\tau} a_{11}(s) d s \neq 0$ ) in $B$ and $x_{1}=L_{3} f_{3}$ define a linear, compact operator $L_{1}: B \rightarrow$ $B$. Then, we have

$$
€\left(f_{1}, f_{2}\right) \equiv\left(L_{1}\left(a_{12} L_{2} f_{2}\right), L_{2} f_{2}\right) .
$$

(b) If $\int_{0}^{\tau} a_{22}(s) d s=0, \int_{0}^{\tau} a_{11}(s) d s \neq 0$, then (29) has exactly one independent solution in $B \times B$.

Lemma 7. Suppose $a \in B$ and $(1 / \tau) \int_{0}^{\tau} a(l) d l=0$. Then, $x_{1}^{\prime}=a x+f(f \in B)$ has a solution $x \in B$ if and only if $(1 / \tau) \int_{0}^{\tau} f(l)\left(\exp \left(-\int_{0}^{l} a(s) d s\right) d l\right)=0$.

By Lemma 7, in its invariant manifold $\widetilde{s}=x(t)+y(t)+z(t)$, system (2) is reduced to a equivalently nonautonomous system as the following:

$$
\begin{gathered}
\dot{x}(t)=-x-\frac{m_{1} x y}{a_{1}+x+a_{2} x^{2}}-\frac{m_{2} x(\widetilde{s}-x-y)}{a_{1}+x+a_{2} x^{2}}, \\
\dot{y}(t)=y\left(\frac{m_{1}(1-q) x}{a_{1}+x+a_{2} x^{2}}-1\right), \\
\Delta x=\tau, \quad t=n \tau, \\
\Delta y=0, \quad t,
\end{gathered}
$$

and if $m_{2}>m_{2}^{*}$, the boundary periodic solution $\left(x_{s}, 0\right)$ is locally asymptotically stable provided with $m_{1}<m_{1}^{*}$ and it is unstable provided with $m_{1}>m_{1}^{*}$; hence $m_{1}^{*}$ practices as a bifurcation threshold. For system (32), we have the following result.

Theorem 8. If $m_{2}>m_{2}^{*}$ and $a_{1}-2 a_{2} x_{s}^{2} \geq 0$ hold, then there exists a constant $\lambda_{0}$, such that $m_{1} \in\left(m_{1}^{*}, m_{1}^{*}+\lambda_{0}\right)$, and there exists a solution $(x, y)$ satisfying $0<x<x_{s}, y>0, z=\widetilde{s}-$ $x-y>0$. Hence, system (2) has a positive $\tau$-periodic solution $(x, y, \widetilde{s}-x-y)$.

Proof. Let $x_{1}=x-x_{s}, x_{2}=y$, and then system (32) becomes

$$
\begin{gathered}
\frac{d x_{1}}{d t}=F_{11}\left(x_{s}, \widetilde{s}\right) x_{1}+x_{2}+g_{1}\left(x_{1}, x_{2}\right), \\
\frac{d x_{2}}{d t}=F_{22}\left(m_{1}, x_{s}\right) x_{2}+g_{2}\left(x_{1}, x_{2}\right),
\end{gathered}
$$

where

$$
\begin{aligned}
F_{11}\left(x_{s}, \widetilde{s}\right)= & \frac{m_{2}\left(\widetilde{s}-x_{s}\right)}{a_{1}-2 a_{2} x_{s}^{2}}-\frac{m_{2} x_{s}\left(a_{1}-2 a_{2} x_{s}^{2}\right)}{\left(a_{1}+x_{s}+a_{2} x_{s}^{2}\right)^{2}} \\
& -1-\frac{m_{2} \widetilde{s} x_{s}\left(1+2 a_{2} x_{s}\right)}{\left(a_{1}+x_{s}+a_{2} x_{s}^{2}\right)^{2}}, \\
F_{12}\left(m_{1}, x_{s}, \widetilde{s}\right)= & \frac{m_{2} x_{s}\left(a_{1}-2 a_{2} x_{s}^{2}\right)}{\left(a_{1}+x_{s}+a_{2} x_{s}^{2}\right)^{2}}+\frac{m_{2} \widetilde{s} x_{s}\left(1+2 a_{2} x_{s}\right)}{\left(a_{1}+x_{s}+a_{2} x_{s}^{2}\right)^{2}} \\
& -\frac{m_{1} x_{s}}{\left(a_{1}+x_{s}+a_{2} x_{s}^{2}\right)^{2}}, \\
F_{22}\left(m_{1}, x_{s}\right)= & \frac{(1-q) m_{1} x_{s}}{\left(a_{1}+x_{s}+a_{2} x_{s}^{2}\right)^{2}}-1 .
\end{aligned}
$$




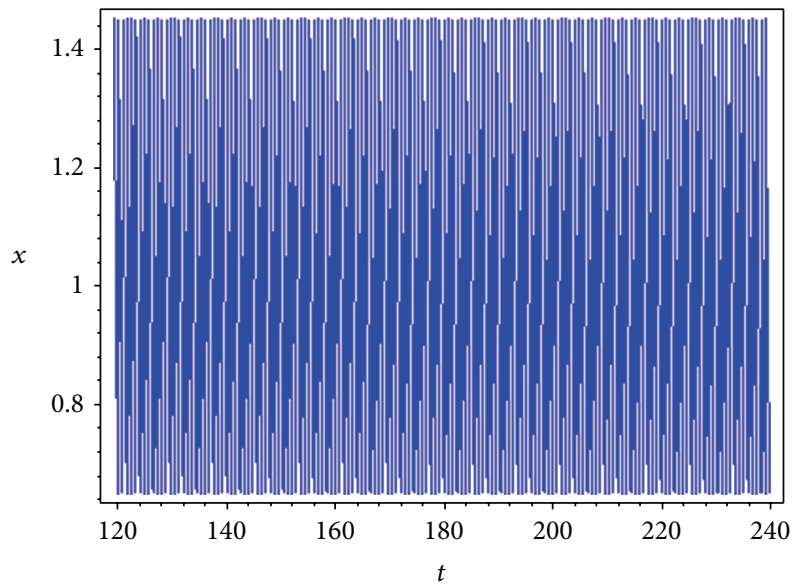

(a)

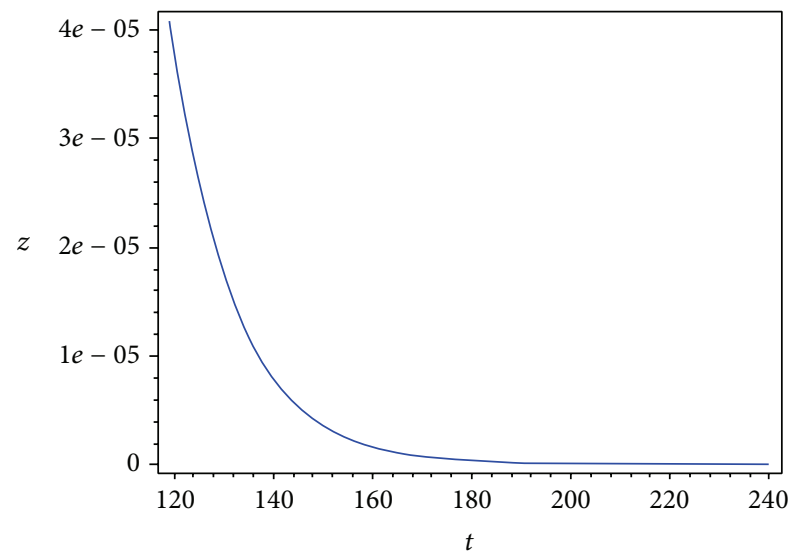

(c)

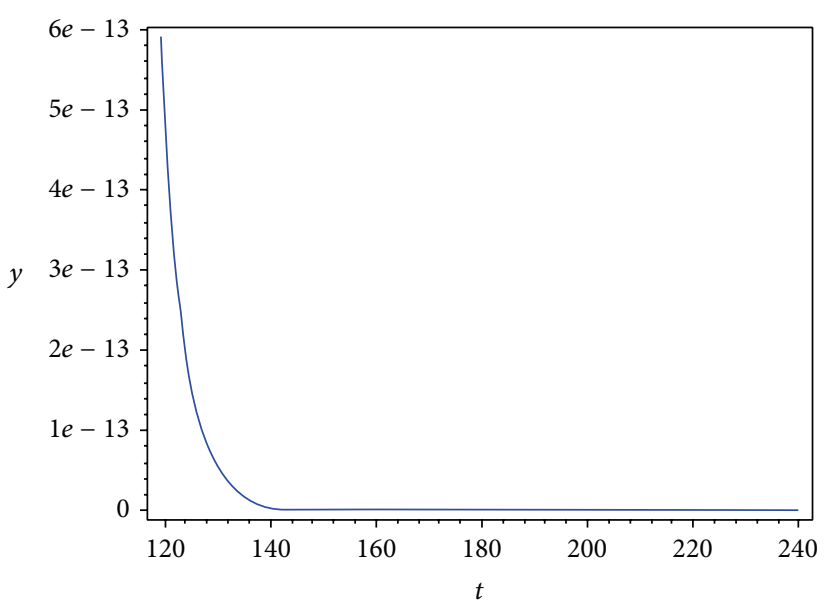

(b)

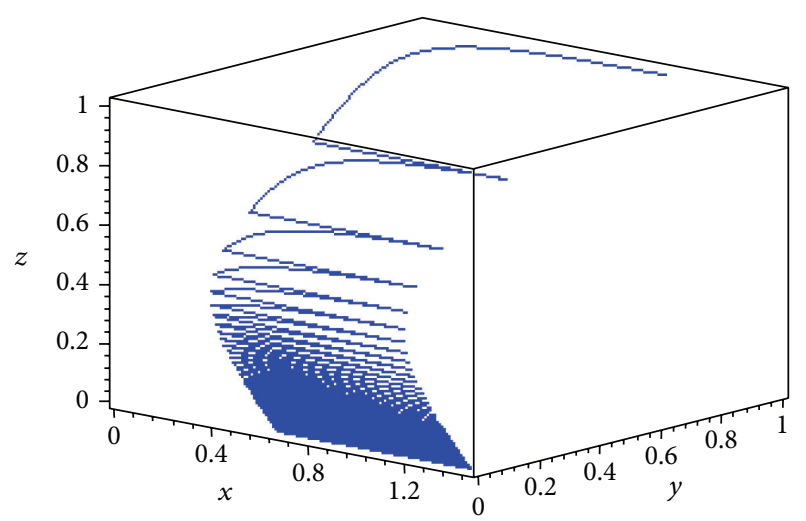

(d)

FIGURE 1: Time series of the system (2) with pulse. $a_{1}=0.2, a_{2}=0.2, m_{1}=1.3, q=0.16$, and $T=0.5$.

We know that $\int_{0}^{\tau}\left(\left((1-q) m_{1} x_{s}(l) /\left(a_{1}+x_{s}(l)+a_{2} x_{s}^{2}\right)^{2}(l)\right)\right)-$ 1) $d l \neq 0$, and, by Lemma 7 , we can equivalently write system (33) as the operator equation

$$
\left(x_{1}, x_{2}\right)=L^{*}\left(x_{1}, x_{2}\right)+G\left(x_{1}, x_{2}\right)
$$

where

$$
\begin{gathered}
G\left(x_{1}, x_{2}\right)=L_{1}\left(F_{12}\left(x_{s}, \widetilde{s}\right) g_{2}\left(x_{1}, x_{2}\right)+g_{1}\left(x_{1}, x_{2}\right),\right. \\
\left.L_{2} g_{2}\left(x_{1}, x_{2}\right)\right) .
\end{gathered}
$$

Here, $L^{*}: B \times B \rightarrow B \times B$ is linear and compact. $G$ : $B \times B \rightarrow B \times B$ is continuous and compact (since $L_{1}$ and $L_{2}$ are compact) and satisfies $G=o\left(\left|\left(x_{1}, x_{2}\right)\right|_{0}\right)$ near $(0,0)$. A nontrivial solution $\left(x_{1}, x_{2}\right) \neq(0,0)$ for some $m_{1}>1$ yields a solution $(x, y)=\left(x_{s}+x_{1}, x_{2}\right) \neq(0,0)$ of system (32). Thus, the existence of the periodic solution of system (2) can be considered as the bifurcation problem of system (35). Now, we apply local bifurcation technique to (35). As is well known, bifurcation can occur only at the nontrivial solution of linearized problem

$$
\left(y_{1}, y_{2}\right)=L^{*}\left(y_{1}, y_{2}\right), \quad m_{1}>0 \text {. }
$$

If $y_{1} \times y_{2} \in B \times B$ is a solution of (37) for some $m_{1}>0$, then $\left(y_{1}, y_{2}\right)$ satisfies

$$
\begin{gathered}
\frac{d y_{1}}{d t}=F_{11}\left(x_{s}, \widetilde{s}\right) y_{1}+F_{12}\left(x_{s}, \widetilde{s}\right) y_{2}, \\
\frac{d y_{2}}{d t}=F_{22}\left(x_{s}\right) y_{2} .
\end{gathered}
$$

By virtue of Lemma 7 (b) and system (38), system (37) has one nontrivial solution in $B \times B$ if and only if $m_{1}^{*}=m_{1}$. Therefore, we obtain one piecewise continuous periodic solution of system (35), which is all nontrivial solutions expect for $\left(m_{1}^{*}, 0,0\right)$. Now, we investigate the solution $\left(m_{1}, x_{1}, x_{2}\right)$ near the bifurcation point $\left(m_{1}^{*}, 0,0\right)$ by expanding $m_{1}$ and $x_{1}, x_{2}$ in a Lyapunov-Schmidt series:

$$
\begin{gathered}
m_{1}=m_{1}^{*}+\lambda \varepsilon+\cdots, \\
x_{1}=x_{11} \varepsilon_{1} x_{12} \varepsilon^{2}+\cdots, \\
x_{2}=x_{21} \varepsilon+x_{22} \varepsilon^{2}+\cdots,
\end{gathered}
$$




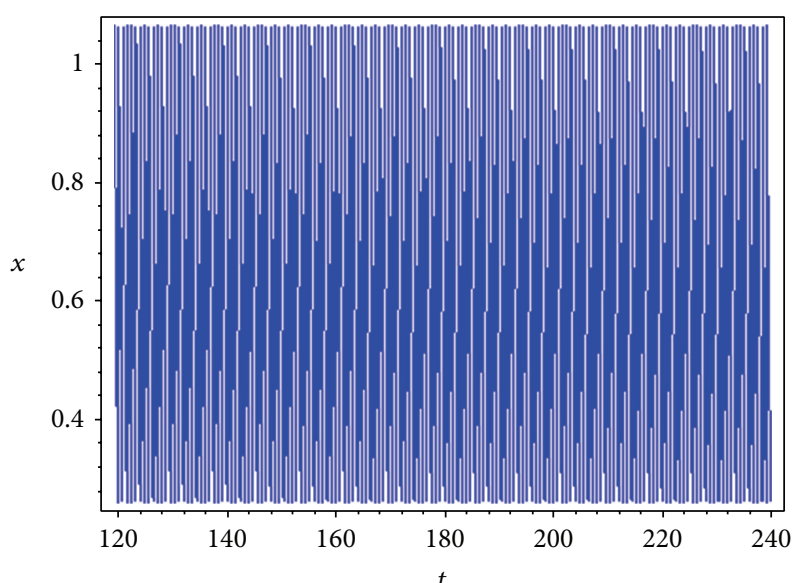

(a)

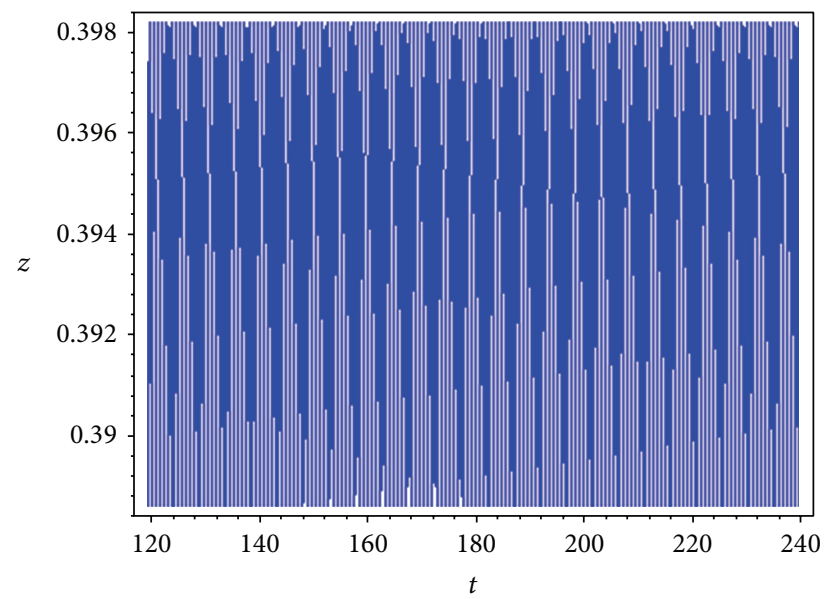

(c)

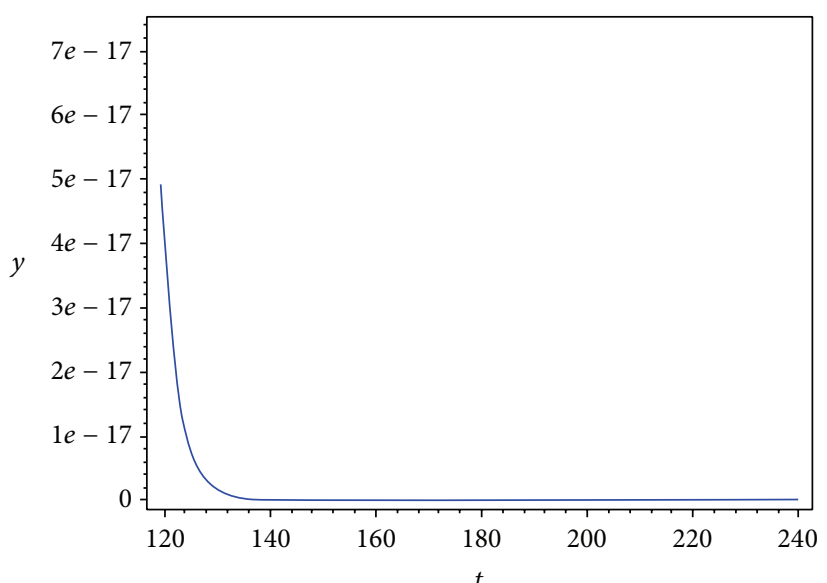

(b)

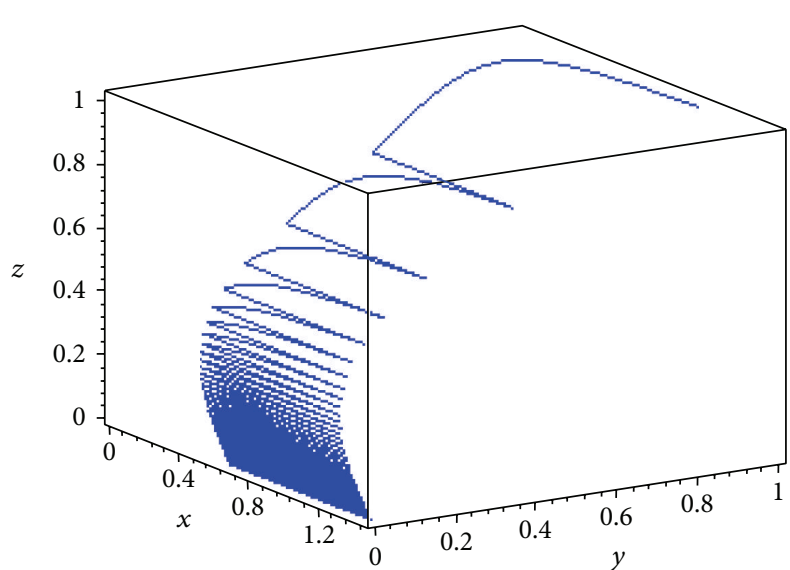

(d)

Figure 2: Time series of the system (2) with pulse. $a_{1}=0.2, a_{2}=0.2, m_{1}=1.3, m_{2}=1.5, q=0.2$, and $T=0.8$.

where $x_{i j} \in B, \varepsilon$ is a small parameter. If we substitute those series into the system (32) and equate coefficients of $\varepsilon$ and $\varepsilon^{2}$, we find that

$$
\begin{aligned}
& x_{11}^{\prime}= F_{11}\left(x_{s}, \widetilde{s}\right) x_{11}+F_{12}\left(m_{1}^{*}, x_{s}, \widetilde{s}\right) x_{21}, \\
& x_{21}^{\prime}=F_{22}\left(m_{1}^{*}, x_{s}\right) x_{21}, \\
& x_{12}^{\prime}=F_{11}\left(x_{s}, \widetilde{s}\right) x_{12}+F_{12}\left(m_{1}^{*}, x_{s}, \widetilde{s}\right) x_{22}, \\
& x_{22}^{\prime}=F_{22}\left(m_{1}^{*}, x_{s}\right) x_{22}+\frac{x_{21}(1-q)}{a_{1}+x_{s}+a_{2} x_{s}^{2}} \\
& \times\left(\lambda+\frac{m_{1}^{*} x_{11}\left(a_{1}-2 a_{2} x_{s}^{2}\right)}{a_{1}+x_{s}+a_{2} x_{s}^{2}}\right) .
\end{aligned}
$$

Thus, $\left(x_{1}, x_{2}\right) \in B \times B$ must exist a solution of (37). Choose the specific solution such as the initial condition $x_{21}(0)=1$, and then we have

$$
x_{21}=\exp \left(\int_{0}^{t}\left((1-q) \frac{m_{1}^{*} x_{s}(l)}{a_{1}+x_{s}(l)+x_{s}^{2}(l)}-1\right) d l\right)>0,
$$

$x_{11}<0$ for all $t$ (since $m_{2}>m_{2}^{*}$ and $(14), \int_{0}^{\tau}\left(\left(m_{2}\left(\widetilde{s}-x_{s}\right) /\left(a_{1}-\right.\right.\right.$ $\left.\left.2 a_{2} x_{s}^{2}\right)\right)-\left(m_{2} x_{s}\left(a_{1}-2 a_{2} x_{s}^{2}\right) /\left(a_{1}+x_{s}+a_{2} x_{s}^{2}\right)^{2}\right)-1-\left(m_{2} \tilde{s} x_{s}(1+\right.$ $\left.\left.\left.2 a_{2} x_{s}\right) /\left(a_{1}+x_{s}+a_{2} x_{s}^{2}\right)^{2}\right)\right) d l=-\int_{0}^{\tau}\left(\left(m_{2} x_{s}\left(a_{1}-2 a_{2} x_{s}^{2}\right) /\left(a_{1}+\right.\right.\right.$ $\left.\left.\left.x_{s}+a_{2} x_{s}^{2}\right)^{2}\right)+\left(m_{2} \widetilde{s} x_{s}\left(1+2 a_{2} x_{s}\right) /\left(a_{1}+x_{s}+a_{2} x_{s}^{2}\right)^{2}\right)\right) d l<0$ implies that Green's function for the first equation in (38) is positive). From Lemma 7, we obtain that

$$
\begin{gathered}
\lambda=-\left(\left(\int_{0}^{\tau} \frac{m_{1}^{*} x_{11}(t) x_{21}(t)\left(a_{1}-a_{2} x_{s}^{2}(t)\right)}{\left(a_{1}+x_{s}(t)+a_{2} x_{s}^{2}(t)\right)^{2}}\right.\right. \\
\quad \times \exp \left(\int_{0}^{t}\left(\frac{(1-q) m_{1}^{*} x_{s}(l)}{a_{1}+x_{s}(l)+a_{2}+x_{s}^{2}(l)}-1\right)\right. \\
d l) d t) \\
\times\left(\int_{0}^{\tau} \frac{x_{21}(t)}{a_{1}+x_{s}(t)+a_{2} x_{s}^{2}(t)}\right.
\end{gathered}
$$




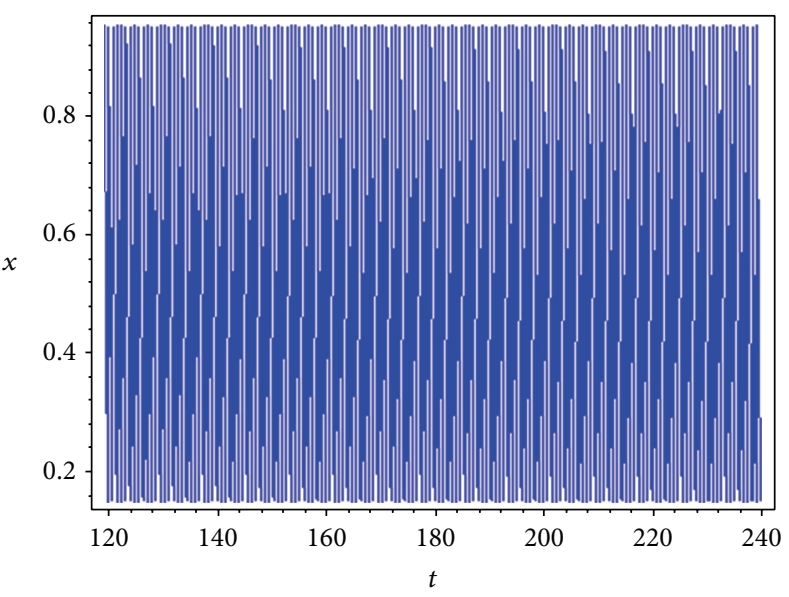

(a)

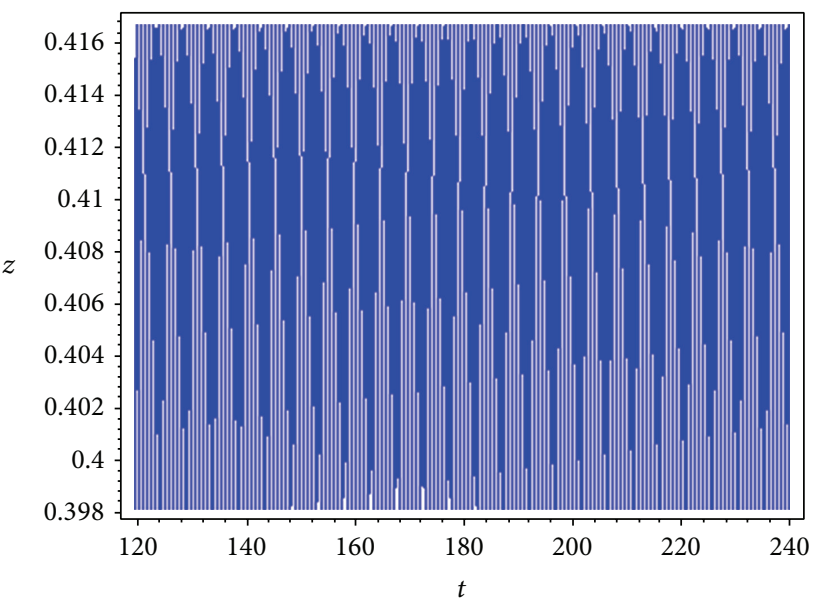

(c)

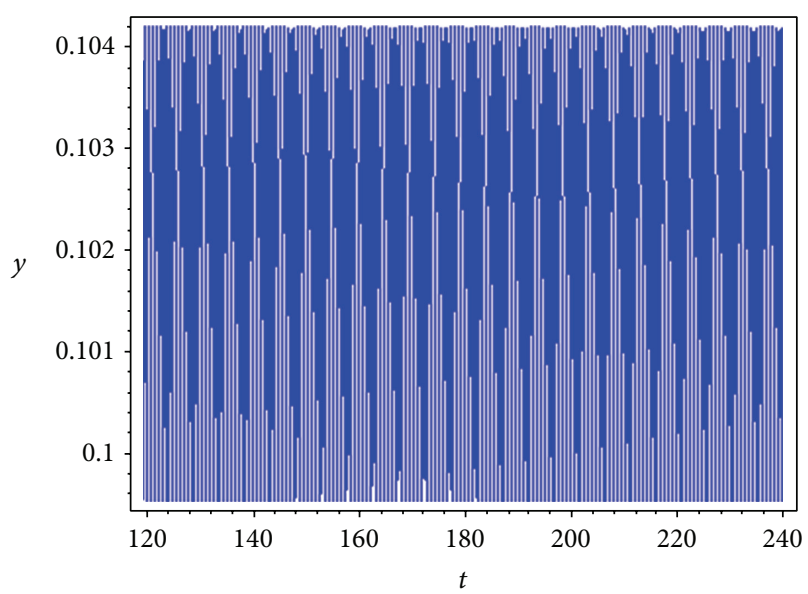

(b)

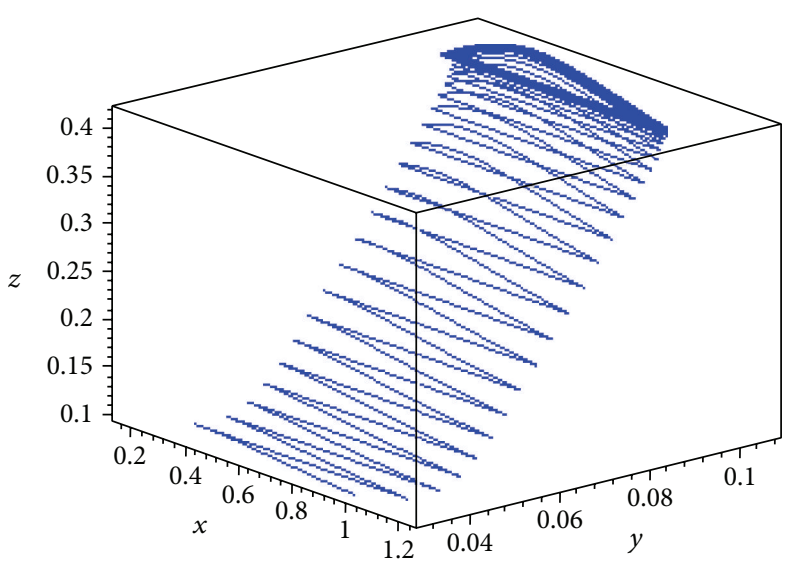

(d)

FIGURE 3: Time series of the system (2) with pulse. $a_{1}=0.2, a_{2}=0.2, m_{1}=2, m_{2}=1.5, q=0.2$, and $T=0.8$.

$$
\begin{gathered}
\times \exp \left(\int_{0}^{t}\left(\frac{(1-q) m_{1}^{*} x_{s}(l)}{a_{1}+x_{s}(l)+a_{2}+x_{s}^{2}(l)}-1\right)\right. \\
\left.d l) d t)^{-1}\right)>0
\end{gathered}
$$

provided with $a_{1}-2 a_{2} x_{s}^{2} \geq 0$, which shows that near the bifurcation point $\left(m_{1}^{*}, 0,0\right)$, there exists a constant $\lambda_{0}$, such that $m_{1} \in\left(m_{1}^{*}, m_{1}^{*}+\lambda_{0}\right)$. Thus, system (29) has a solution $(x, y) \in(B \times B), y>0$. Next, we have only to show that $x=x_{1}+x_{s}>0$ for all $t>0$; that is, if $\lambda_{0}$ is small, then $x$ is near $x_{s}$ in the sup norm of $B$. Since $x_{s}$ is bounded away from zero, so is $x$. According to Theorem 5 , for system (2), $x$ is near $x_{s}$, which implies that $z$ is near $z_{s}$. We notice that the period of the periodic solution $(x, y)$ is $\tau$; therefore, $z=\widetilde{s}-x-y>0$ is piecewise continuous and $\tau$-periodic. The proof is completed.

\section{Numerical Simulations}

In order to justify our theoretic results, we will give some numerical simulations.

Let the parameters of system (2) be $a_{1}=0.2, a_{2}=$ $0.2, m_{1}=1.3, m_{2}=1.3, q=0.16$, and $T=0.8$. By computing, $m_{2}^{*}=1.404>m_{2}=1.3$ is obtained. From Theorem 5, we know that if $m_{2}^{*}>m_{2}$, system (2) has a unique globally asymptotically stable boundary $\tau$-periodic solution $(\widetilde{s}(t), 0,0)$, which is shown in Figure 1.

Suppose that $a_{1}=0.2, a_{2}=0.2, m_{1}=1.3, m_{2}=1.5$, $q=0.2$, and $T=0.8$. By computing, we have $m_{2}^{*}=1.410<$ $m_{2}=1.5, m_{1}^{*}=1.763>m_{1}=1.3$. According to Theorem 5, for $m_{2}>m_{2}^{*}$ and $m_{1}<m_{1}^{*}$, system (2) has a unique globally asymptotically stable boundary $\tau$-periodic solution $\left(x_{s}(t)\right), 0, z_{s}(t)$, which is demonstrated in Figure 2 .

Set $a_{1}=0.2, a_{2}=0.2, m_{1}=2, m_{2}=1.5, q=0.2$, and $T=$ 0.8 . By computing, we obtain $m_{1}^{*}=1.763<m_{1}=2, m_{2}^{*}=$ $1.410<m_{2}=1.5$. From Theorem 5, when $m_{2}>m_{2}^{*}$ and $m_{1}>m_{1}^{*}$, system (2) exists a positive periodic solution, which is simulated in Figure 3. 


\section{Discussion}

In this paper, we have investigated a competitive model of plasmid-bearing and plasmid-free organisms in a pulsed chemostat. It is important in biotechnology for the study of plasmid stability where the effects of plasmid loss in genetically altered organisms (the plasmid-free organism is presumably the better competitor) are investigated. Firstly, we find the invasion threshold of the plasmid-free organism, which is $m_{2}^{*}=\left(\tau / \int_{0}^{\tau}\left(\widetilde{s}(l) /\left(a_{1}+\widetilde{s}(l)+a_{2} \widetilde{s}^{2}(l)\right)\right) d l\right)$. If $m_{2}<$ $m_{2}^{*}$, the microorganism-free periodic solution $(\widetilde{s}(0), 0,0)$ is globally asymptotically stable, which is simulated in Figure 1. If $m_{2}>m_{2}^{*}$, then the plasmid-free organism begins to invade the system. Furthermore, we have proved that if $m_{2}>m_{2}^{*}$, there exists $m_{1}^{*}=\left(\tau / \int_{0}^{\tau}\left((1-q) x_{s}(l) /\left(a_{1}+x_{s}(l)+a_{2} x_{s}^{2}(l)\right)\right) d l\right)$ as the invasion threshold of the plasmid-bearing; that is to say, if $m_{1}<m_{1}^{*}$ the boundary periodic solution $\left(x_{s}(t), 0, z_{s}(t)\right)$ is globally asymptotically stable (see Figure 2) and if $m_{1}>$ $m_{1}^{*}$, the solution $\left(x_{s}(t), 0, z_{s}(t)\right)$ is unstable. Lastly by using standard techniques of bifurcation theory, we prove that if $m_{2}>m_{2}^{*}$ and $a_{1}-a_{2} x_{s}^{2} \geq 0$, the system has a positive $\tau$-periodic solution, which is shown in Figure 3.

System (2) compared with other literatures is more interesting since it can be used to model the excess substrate inhibition in the process of the genetically modified fermentation. We show that the plasmid-bearing and plasmid-free can coexist on a periodic solution. The analysis indicates that the change of one organism into another can complicate the dynamics of system (2) since the plasmid-bearing concentration is relevant to the substrate concentration and the substrate concentration can be controlled by setting the period of impulsive input. However, how to find the optimal period of impulsive input makes microorganisms reach the maximum production by genetically altered biotechnology, which is a challenging problem to solve and we will leave it for the future.

\section{Conflict of Interests}

The authors declare that there is no conflict of interests regarding the publication of this paper.

\section{Acknowledgments}

This work is supported by the National Natural Science Foundation of China (no. 11371164), NSFC-Talent Training Fund of Henan (no. U1304104), the young backbone teachers of Henan (no. 2013GGJS-214), and Henan Science and Technology Department (nos. 132300410084 and 132300410250).

\section{References}

[1] F. Mazenc, M. Malisoff, and P. De Leenheer, "On the stability of periodic solutions in the perturbed chemostat," Mathematical Biosciences and Engineering, vol. 4, no. 2, pp. 319-338, 2007.

[2] F. Mazenc, M. Malisoff, and J. Harmand, "Stabilization and robustness analysis for a chemostat model with two species and monod growth rates via a Lyapunov approach," in Proceedings of the 46th IEEE Conference on Decision and Control, pp. 39333938, New Orleans, La, USA, December 2007.

[3] F. Mazenc, M. Malisoff, and J. Harmand, "Further results on stabilization of periodic trajectories for a chemostat with two species," IEEE Transactions on Automatic Control, vol. 53, pp. 66-74, 2008.

[4] H. Nie and J. H. Wu, "Positive solutions of a competition model for two resources in the unstirred chemostat," Journal of Mathematical Analysis and Applications, vol. 355, no. 1, pp. 231242, 2009.

[5] S. L. Yuan, W. G. Zhang, and M. A. Han, "Global asymptotic behavior in chemostat-type competition models with delay," Nonlinear Analysis. Real World Applications, vol. 10, no. 3, pp. 1305-1320, 2009.

[6] O. Tagashira, "Permanent coexistence in chemostat models with delayed feedback control," Nonlinear Analysis. Real World Applications, vol. 10, no. 3, pp. 1443-1452, 2009.

[7] M. I. Nelson and H. S. Sidhu, "Analysis of a chemostat model with variable yield coefficient," Journal of Mathematical Chemistry, vol. 38, no. 4, pp. 605-615, 2005.

[8] M. I. Nelson and H. S. Sidhu, "Analysis of a chemostat model with variable yield coefficient: tessier kinetics," Journal of Mathematical Chemistry, vol. 46, no. 2, pp. 303-321, 2009.

[9] H. L. Smith and P. Waltman, The Theory of the Chemostat, vol. 13 of Cambridge Studies in Mathematical Biology, Cambridge University Press, Cambridge, UK, 1995.

[10] Z. Y. Xiang and X. Y. Song, "A model of competition between plasmid-bearing and plasmid-free organisms in a chemostat with periodic input," Chaos, Solitons \& Fractals, vol. 32, no. 4, pp. 1419-1428, 2007.

[11] X. Shi, X. Song, and X. Zhou, "Analysis of a model of plasmidbearing, plasmid-free competition in a pulsed chemostat," Advances in Complex Systems, vol. 9, no. 3, pp. 263-276, 2006.

[12] R. Pal, D. Basu, and M. Banerjee, "Modelling of phytoplankton allelopathy with Monod-Haldane-type functional responsea mathematical study," BioSystems, vol. 95, no. 3, pp. 243-253, 2009.

[13] J. M. Cushing, "Periodic time-dependent predator-prey systems," SIAM Journal on Applied Mathematics, vol. 32, no. 1, pp. 82-95, 1977. 


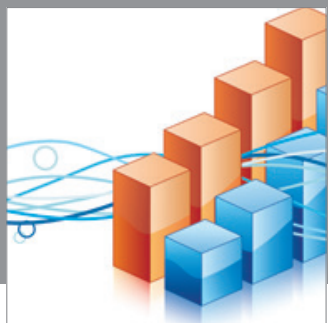

Advances in

Operations Research

mansans

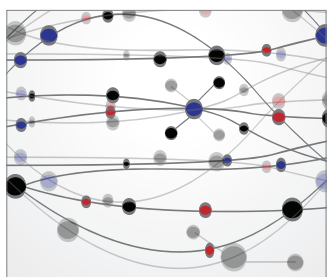

The Scientific World Journal
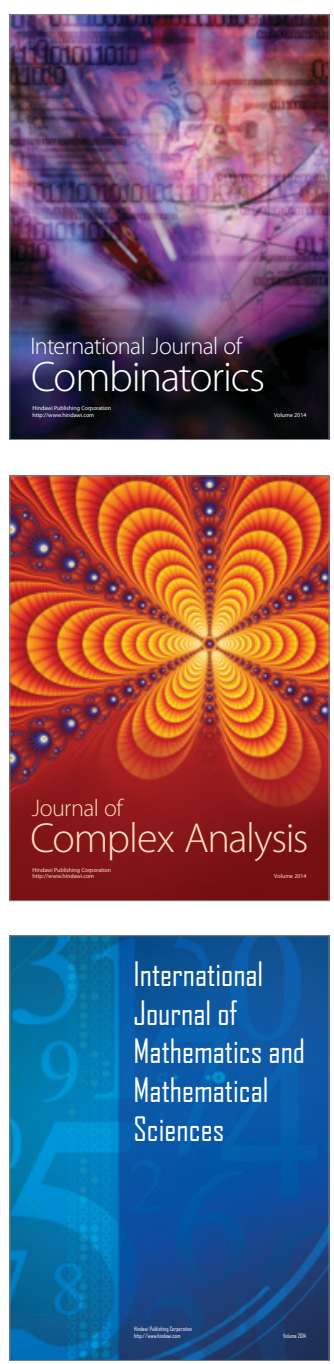
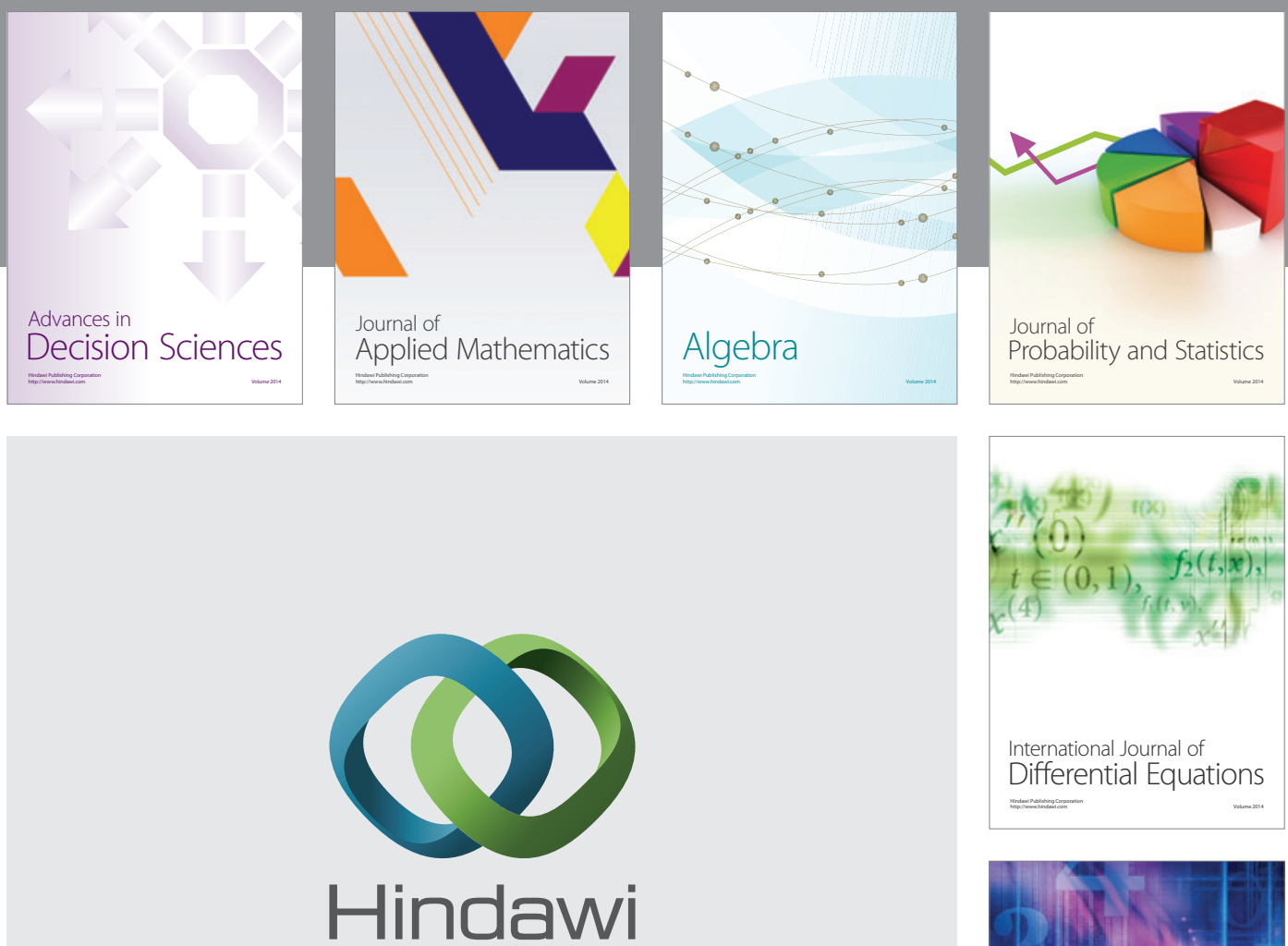

Submit your manuscripts at http://www.hindawi.com
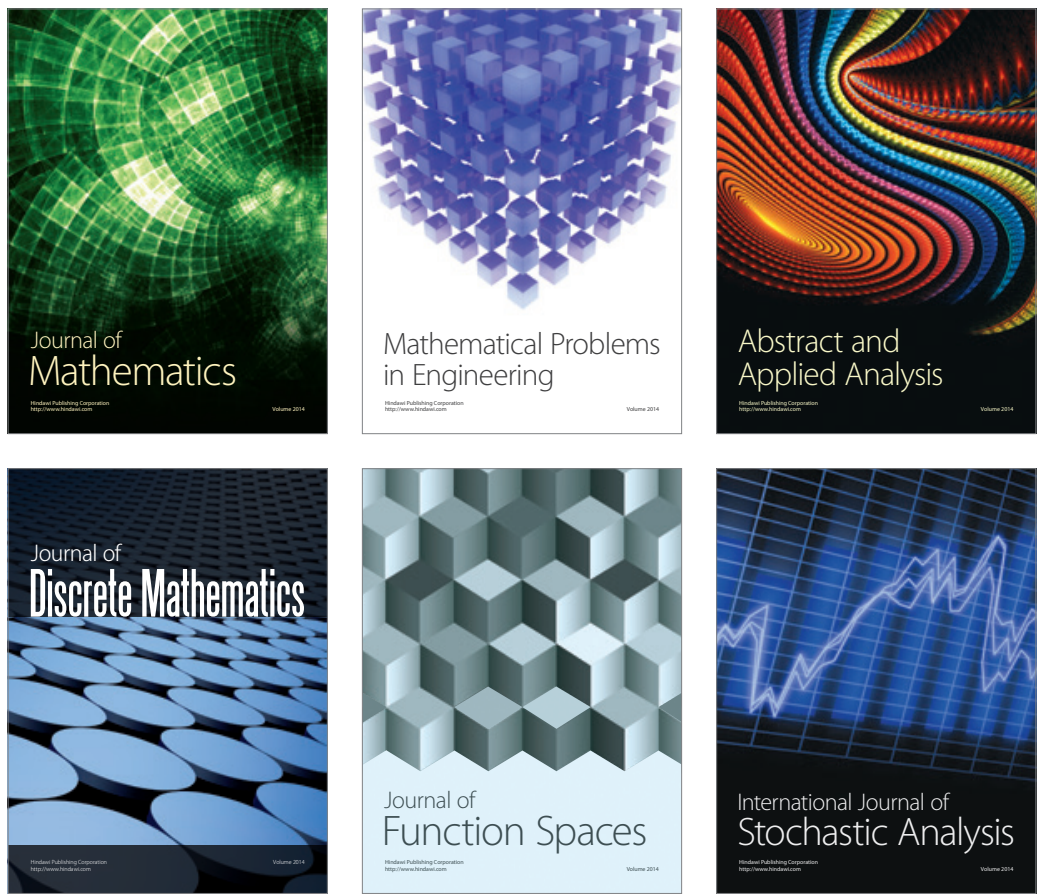

Journal of

Function Spaces

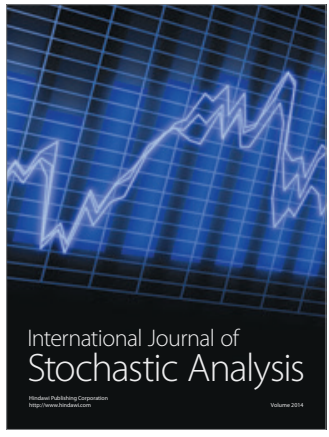

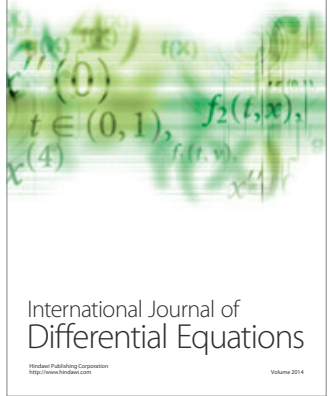
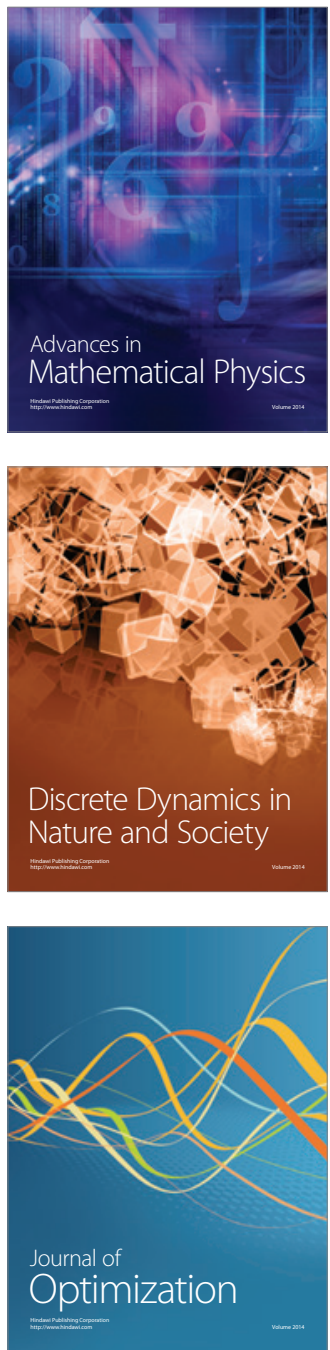